DFTUZ $98 / 18$

\title{
Baryonic thermal fluctuations in finite temperature QCD
}

\author{
Vicente Azcoiti and Angelo Galante \\ Departamento de Física Teórica, Facultad de Ciencias, Universidad de Zaragoza, \\ 50009 Zaragoza (Spain).
}

\begin{abstract}
We show how, contrary to physical intuition, thermal fluctuations of physical states having a non-vanishing baryonic number can be fully neglected in the thermodynamics of $Q C D$ at any physical temperature. We also discuss on the consistency between our results and the CPT theorem. The last part of this article is devoted to comment some interesting physical features which follow from this result.
\end{abstract}


The partition function of a quantum field theory at zero temperature is dominated by the contribution of the vacuum state, i.e., the eigenstate of the quantum hamiltonian having minimum energy. By increasing the physical temperature, contributions to the free energy density of excited states become more and more relevant and in the infinite temperature limit the system fluctuates randomly and all the states have the same probability, no matter what energy they have.

In $Q C D$, the gauge theory describing the strong interaction of particles, the $S U(3)$ gauge symmetry of the model implies that physical states are made up of a multiple of three number of quarks. Hence we have baryons and mesons in the spectrum of this model and a natural expectation is that thermal fluctuations of both kind of particles will be relevant at finite temperature. Notwithstanding that we will show in this letter how thermal fluctuations of physical states having a non-vanishing baryonic charge can be fully neglected in the thermodynamics of $Q C D$ at any physical temperature [1]. The last part of this article will be devoted to discuss some interesting physical features which follow from this result.

\section{Analytical results}

Due to a global $\mathrm{U}(1)$ symmetry of $Q C D$, the vector current $\bar{\psi} \gamma_{\nu} \psi$ is a conserved current. The integrated fourth component of this current

$$
N=\int d^{3} x \bar{\psi}(x) \gamma_{0} \psi(x)
$$

defines a dynamical invariant, the baryonic charge or quark number operator. 
After quantization the quark number operator commutes with the hamiltonian and physical states can therefore be characterized by its energy and baryonic charge. Hence the Hilbert space of physical states can be decomposed as a direct sum of Hilbert subspaces, each one of them corresponding to a given eigenvalue of the quark number operator.

Taking into account this decomposition of the Hilbert space of physical states we can write the partition function of $Q C D$ at finite temperature

$$
Z=\operatorname{Tr}\left(e^{-\frac{H}{T}}\right)
$$

as a sum of canonical partition functions

$$
Z=\sum_{k} \operatorname{Tr}_{k}\left(e^{-\frac{H}{T}}\right)
$$

each one of the $\operatorname{Tr}_{k}\left(e^{-\frac{H}{T}}\right)$ being the partition function computed in the baryonic sector of the Hilbert space corresponding to a given eigenvalue $k$ of the quark number operator.

In order to establish the connection between this hamiltonian approach and the path integral formulation in the lattice regularization scheme, and to identify in the latter formalism what are the different baryonic sector contributions to the partition function, the simplest way is to introduce a chemical potential $\mu$ since it couples directly with the quark number operator $N$. The Grand Canonical Partition Function $(G C P F)$ is given then by the following expression 


$$
Z_{G C}=\operatorname{Tr}\left(e^{-\frac{1}{T}(H-\mu N)}\right)
$$

Using now the previous decomposition of the Hilbert space in different baryonic sectors we can write the $G C P F$ as

$$
Z_{G C}=\sum_{k} e^{\frac{\mu}{T} k} \operatorname{Tr}_{k}\left(e^{-\frac{H}{T}}\right),
$$

which is the standard decomposition of the $G C P F$ as a sum of canonical partition functions. Setting the chemical potential $\mu=0$ we recover expression (3).

The standard way to introduce a chemical potential in lattice regularized $Q C D$ is to multiply all temporal link variables pointing forward (backward) by $e^{\mu}\left(e^{-\mu}\right)$ [2], [3]. The previous decomposition of the GCPF as a sum of canonical partition functions corresponds on the lattice to the Polyakov loop expansion of the partition function

$$
Z_{G C}=\bar{a}_{3 V_{x}} e^{3 V_{x} \frac{\mu}{T}}+\bar{a}_{3\left(V_{x}-1\right)} e^{3\left(V_{x}-1\right) \frac{\mu}{T}}+\ldots . .+\bar{a}_{0}+\ldots \ldots+\bar{a}_{-3 V_{x}} e^{-3 V_{x} \frac{\mu}{T}}
$$

where $V_{x}$ is the lattice spatial volume, the temperature $T$ is the inverse lattice temporal extent and the indices in the sum are numbers of vanishing triality as a consequence of the fact that physical states are made up of a multiple of three number of quarks. This last property can also be derived from the invariance of the pure gauge action under $Z(3)$ global transformations.

The relation between the integrated coefficients $\bar{a}_{k}$ in (6) and the contribution $a_{k}(U)$ from a single gauge configuration is 


$$
\bar{a}_{k}=\int[D U] a_{k}(U) e^{-\beta S_{G}(U)} .
$$

where $S_{G}(U)$ is the standard pure gauge Wilson action, $\beta$ the square of the inverse gauge coupling and $a_{k}(U)$ is the contribution to the determinant of the Dirac-Kogut-Susskind operator containing a net number of Polyakov loops equal to $k$.

Expression (6) for the partition function has been written assuming the Kogut-Susskind regularization for the fermionic degrees of freedom. Under such a regularization, $3 V_{x}$ is the maximum number of Polyakov loops which can appear in the fermion determinant for a lattice with $V_{x}$ spatial points. Equivalently $3 V_{x}$ is also the maximum number of three-colored quarks of the Kogut-Susskind type we can put in a finite discrete space with $V_{x}$ points following the Fermi-Dirac statistics.

From the comparison of expressions (5) and (6) we can conclude that the coefficient $\bar{a}_{k}$ is equal to the canonical partition function $\operatorname{Tr}_{k}\left(e^{-\frac{H}{T}}\right)$ computed in the baryonic sector of the Hilbert space having a baryonic number equal to $\frac{k}{3}$ [2].

In order to analyze the relevance of the different coefficients $\bar{a}_{k}$ for different values of the baryon number $\frac{k}{3}$, we will consider the fermion determinant for a given gauge configuration in presence of an imaginary chemical potential $\mu=\frac{i \eta}{L_{t}}[\mathbb{1}]$

$$
\operatorname{det} \Delta(\eta, U)=\sum_{k} a_{k}(U) e^{i k \eta}
$$


where the sum in (8) is over all integer numbers between $-3 V_{x}$ and $3 V_{x}$.

The antihermiticity and $\gamma_{5}$ anticommuting properties of the massless Dirac operator are preserved after the inclusion of an imaginary chemical potential. It follows that the positivity of the fermion determinant is also preserved and

$$
\operatorname{det} \Delta(\eta, U) \geq 0
$$

for any gauge configuration and any $\eta$. This property of positivity will be fundamental in order to derive bounds for the partition functions $\bar{a}_{k}$ which will allow us to get interesting conclusions on the relevance of the different baryonic sectors. In a similar way Vafa and Witten used the positivity of the effective fermionic action in vector-like theories to get bounds which drive to the impossibility to break spontaneously vector-like global symmetries as isospin or baryon number conservation [4].

A simple Fourier transformation in (8) gives

$$
a_{k}(U)=\frac{1}{2 \pi} \int e^{-i k \eta} \operatorname{det} \Delta(\eta, U) d \eta
$$

and, making use of the positivity relation (9), the following inequalities can be derived

$$
a_{0}(U) \geq 0, \quad\left|a_{k}(U)\right| \leq a_{0}(U) \quad \forall k .
$$

These relations, which hold for any gauge configuration, imply for the integrated coefficients or canonical partition functions $\bar{a}_{k}$ the inequalities 
$\bar{a}_{k} \leq \bar{a}_{0}$, i.e.,

$$
\frac{\operatorname{Tr}_{k}\left(e^{-\frac{H}{T}}\right)}{\operatorname{Tr}_{0}\left(e^{-\frac{H}{T}}\right)} \leq 1
$$

The relation (12) between canonical partition functions induces interesting physical consequences in the thermodynamics of this model. To analyze them let us consider the free energy density,

$$
f=\frac{T}{V_{x}} \log Z
$$

Using the obvious relation $\bar{a}_{k}=\bar{a}_{-k}$ the partition function at zero chemical potential can be written as

$$
Z=\bar{a}_{0}\left(1+\frac{2 \bar{a}_{3}}{\bar{a}_{0}}+\ldots \ldots \ldots+\frac{2 \bar{a}_{3 V_{x}}}{\bar{a}_{0}}\right)
$$

a relation which gives for the free energy density the following expression

$$
f=\frac{T}{V_{x}} \log \bar{a}_{0}+\frac{T}{V_{x}} \log \left(1+\frac{2 \bar{a}_{3}}{\bar{a}_{0}}+\ldots \ldots \ldots+\frac{2 \bar{a}_{3 V_{x}}}{\bar{a}_{0}}\right) .
$$

The first contribution in (15) is the free energy density of the vanishing baryon number sector. The second term contains the contribution of all the other non-vanishing baryon number sectors. Relation (12) implies that the only contribution surviving the thermodynamical limit is the first one. The free energy density of $Q C D$ at any physical temperature $T$ is given therefore by

$$
f=\frac{T}{V_{x}} \log \bar{a}_{0}=\frac{T}{V_{x}} \log \left(\operatorname{Tr}_{0}\left(e^{-\frac{H}{T}}\right)\right) .
$$


The previous expression implies that all baryonic thermal fluctuations can be neglected in the thermodynamics of $Q C D$ (at zero chemical potential). Notwithstanding that, this result does not necessarily imply that the probability $p_{k}$ to get a sector with non-vanishing baryon number vanishes. What happens is that if $\frac{p_{k}}{p_{0}}=\frac{\bar{a}_{k}}{\bar{a}_{0}}$ is a non-vanishing number in the infinite volume limit, sector $k$ has the same free energy density as sector 0 and therefore the same thermodynamical properties.

The CPT theorem [5] states that the vacuum state is CPT invariant. Since the baryon number density is an order parameter for the CPT symmetry, the theorem enforces the probability distribution function of the baryon number density to be a $\delta$ function centered at the origin. Our results are therefore consistent with the CPT theorem but also give some further information: the partition function of QCD at any physical temperature can be computed not only in the vanishing baryonic density sector but also in the vanishing baryon number sector i.e., all baryonic thermal fluctuations can be neglected.

This is something different from the standard statistical mechanics result that relative fluctuations go to zero as the thermodynamical limit is approached. In fact we state that all properties related to the free energy density can be derived considering the zero baryon number sector only. Clearly the result does not apply to the derivatives respect to the chemical potential i.e., the baryonic susceptibility at zero chemical potential can not be evaluated from the vanishing baryon number sector only. 


\section{Some interesting physical implications}

Once we have demonstrated that thermal fluctuations of physical states with non-vanishing baryon number can be neglected in the thermodynamics of $Q C D$, we will devote the last part of this article to the discussion of some interesting physical implications of this result.

i) The first interesting feature is the possibility of analyzing the deconfining phase transition at finite temperature by means of an order parameter. The mean value of the Polyakov loop or Wilson line [6] is related to the energy of an isolated quark in the fluctuating background at a given physical temperature $T$. The standard wisdom is that the mean value of this operator is not an order parameter in the full theory with dynamical fermions since the determinant of the Dirac operator, which appears in the integration measure, breaks explicitly the Polyakov symmetry. The possibility of using the mean value of the Polyakov loop as an exact order parameter in the full theory with dynamical fermions, which was pointed out in early works [0], [8], becomes apparent in the formalism here developed. In fact $a_{0}(U)$, the contribution to the fermion determinant containing a vanishing net number of Polyakov loops, is invariant under Polyakov transformations. Even more, $a_{0}(U)$ is also independent of the boundary conditions for the fermion field in the temporal direction. Periodic or antiperiodic boundary conditions give rise to the same thermodynamical properties.

ii) Theoretical prejudices based on simple models tell us that the high density phase of $Q C D$ should be similar to the high temperature phase in 
the sense that both phases would be described by a free quark-gluon plasma. However the physical background in the two limiting cases, high temperature at vanishing chemical potential and high baryonic density at vanishing temperature, shows important differences. In the first case the ground state is dominated by thermal fluctuations of physical states with vanishing baryon number whereas in the second case, physical states with a high baryonic density are the only relevant contributions. Therefore even if the theoretical scenario of a quark-gluon plasma phase describes actually the two phases, we should expect important phenomenological differences between the two limiting extremes of the $(\mu, T)$ phase diagram.

iii) The results here obtained allow also to solve the inconsistencies found in quenched $Q C D$ simulations at finite temperature. As it was shown a few time ago [9], [10] quenched simulations of $Q C D$ at finite temperature and in the broken deconfined phase gave inconsistent results for the chiral condensate and different values for the critical temperature of the chiral transition were found, depending on the phase of the Polyakov loop in which the dynamics settles. These results, which put in question the validity of the quenched approximation, are not unexpected. In fact the integration measure is invariant under Polyakov transformations in this approximation but the chiral condensate operator is not. A value of the chiral condensate depending on the $Z(3)$ vacuum in which the dynamics settles in the broken phase is a rather expected result on physical grounds. However, as previously shown, the free energy density of $Q C D$ can be computed neglecting all the contributions to the partition function of non-vanishing baryon number sectors. We can take 
$a_{0}(U)$ instead of det $\Delta(U)$ in the integration measure of full $Q C D$ and consistently the logarithmic mass derivative of $a_{0}(U)$ instead of the trace of the inverse Dirac operator as chiral condensate operator. Within this prescription, the chiral order parameter is invariant under Polyakov transformations and its mean value is therefore the same in each of the three possible $Z(3)$ vacuums of the quenched broken phase.

iv) Another topic in which the results here reported could give rise to an interesting improvement is the one concerning finite size effects and MonteCarlo convergence in numerical simulations of finite temperature $Q C D$. As previously discussed, most of the contributions of the different baryon number sectors, specially those corresponding to a non-vanishing baryon number density, will have a vanishing probability in the thermodynamical limit. They are pure finite size effects and a natural expectation is that with our prescription of taking $a_{0}(U)$ instead of det $\Delta(U)$ in the integration measure, we will approach the thermodynamical limit faster. But even if some of these contributions have a non-vanishing probability, our prescription of taking $a_{0}(U)$ in the integration measure should improve the Monte-Carlo convergence in numerical simulations of finite temperature $Q C D$. In fact the coefficient $a_{0}(U)$ has more symmetries than the determinant of the full Dirac operator and, as we have learned from the $G C P F$ computation in the investigations of $Q C D$ at finite density [11], [12] $a_{0}(U)$ is less fluctuating than $\operatorname{det} \Delta(U)$. This last feature is the manifestation of what is known as the sign problem in finite density $Q C D$ [13], a problem that has delayed enormously the progress in this field. 
The central coefficient $a_{0}(U)$ is positive definite for any gauge configuration but unfortunately this is not the case for almost all the other coefficients. Most of them fluctuate violently with a rather constant absolute value but with an almost randomly distributed phase. Things here are very similar to what happens in the unpleasant situation we find when try to compute a small number by averaging numbers which are several orders of magnitude larger than their mean value. We would need a prohibitive statistics, increasing exponentially with the lattice volume in the GCPF computations [12], [13], to get significant numbers. The contribution of most of the non-vanishing baryon number sectors to the partition function of finite temperature $Q C D$ at actual statistics behaves therefore like a noise, and a natural expectation is that convergence of any Monte-Carlo procedure should improve by killing this noise.

The exact computation of $a_{0}(U)$ is very hard. The best way at present is through the construction of the quark propagator matrix [14] and requires the exact diagonalization of a non hermitian matrix for each gauge configuration. However, as follows from the analysis here developed, there is a simpler way to implement things in practical standard simulations of $Q C D$ at finite temperature. It consists in the introduction of an extra abelian degree of freedom $e^{i \eta}$ coupled to all temporal links and having a dynamical character. The integration over this new dynamical degree of freedom will kill all the undesidered contributions to the partition function. 


\section{Wilson fermions}

We have shown in the previous sections of this paper how the thermodynamics of QCD, when regularized in a space-time lattice and using staggered fermions, is controlled by the contribution to the fermion determinant with no net number of Polyakov loops, i.e., by the thermal fluctuations of physical states with vanishing baryon number.

The two main ingredients to get this result are the conservation of baryonic charge in QCD and the inequalities (12) which tell us that the partition function at fixed baryon number reaches its maximum value in the Hilbert subspace corresponding to zero baryon number. The first one of the two ingredients is independent of the lattice regularization for the Dirac operator. The second one however is based on the positivity of the determinant of the Dirac operator for any gauge configuration and any value of the extra-abelian degree of freedom $e^{i \eta}$.

Since we have made use of the hermiticity and chiral properties of the Dirac-Kogut-Susskind operator, it is natural to ask whether our result applies to any fermion regularization or rather it is related to the presence-absence of the chiral anomaly.

Let us say from the beginning that even if we have not yet a definite answer to this question, there are strong indications suggesting that the chiral anomaly does not play any relevant role here. These indications come from the analysis of the properties of the fermion determinant for Wilson fermions. As well known, the Dirac-Wilson operator $\Delta$ can be written as 


$$
\Delta=I-\kappa M
$$

where $\kappa$ is the hopping parameter and the matrix $\mathrm{M}$ verifies the following chiral relation

$$
\gamma_{5} M \gamma_{5}=M^{+}
$$

Equation (18) implies that if $\lambda$ is eigenvalue of $M, \lambda^{*}$ is also eigenvalue of M, i.e., the fermion determinant is always real. However it could be negative and in fact this unpleasant situation has been found for some gauge configurations in numerical simulations of the Schwinger model done in the unphysical strong coupling region [15]. However the unitary character of the gauge group implies that all the eigenvalues are upper bounded by the relation

$$
|\lambda| \leq 8
$$

which implies that for $\kappa \leq 1 / 8$, det $\Delta \geq 0$. It is easy to verify that under the previous condition $\kappa \leq 1 / 8$, the positivity of $\operatorname{det} \Delta$ also holds in the presence of the extra-abelian degree of freedom previously introduced.

In other words, all the results of this paper can be extended in a straightforward way to Wilson fermions if we impose the restriction $\kappa \leq 1 / 8$, i.e., the hopping parameter region associated to a positive bare fermion mass. 


\section{Acknowledgements}

This work has been partially supported by CICYT (Proyecto AEN971680). The authors thank also INFN for financial support. 


\section{References}

[1] V. Azcoiti, Theory of Elementary Particles ( Proceedings of the 31st International Symposium Ahrenshoop) WILEY-VCH (1997) 284.

[2] J.B. Kogut, H. Matsuoka, M. Stone, H.W. Wyld, S. Shenker, J. Shigemitsu, D.K. Sinclair, Nucl. Phys. B225 [FS9] (1983) 93.

[3] P. Hasenfratz, F. Karsch, Phys. Lett. 125B (1983) 308.

[4] C. Vafa, E. Witten, Nucl. Phys. B234 (1984) 173.

[5] F. Streater, A.S. Wightman, PCT, Spin and Statistics and All That, Benjamin, New York (1964).

[6] A.M. Polyakov, Phys. Lett. B72 (1977) 477.

[7] M. Oleszczuk and J. Polonyi, Ann. of Phys. 227 (1993) 76.

[8] M. Faber, O.A. Borisenko, S. Mashkevich, G.M. Zinovjev. Nucl. Phys. B (Proc. Suppl.) 42 (1995) 484; M. Faber, O.A. Borisenko, G.M. Zinovjev. Nucl. Phys. B444 (1995) 563.

[9] S. Chandrasekharan, N. Christ, Nucl. Phys. B (Proc. Suppl.) 47 (1996) 527.

[10] M.A. Stephanov, Phys. Lett. B375 (1996) 249.

[11] I.M. Barbour, S.E. Morrison, E.G. Klepfish, J.B. Kogut, M.P. Lombardo, Phys. Rev. D56 (1997) 7063. 
[12] R. Aloisio, V. Azcoiti, G. Di Carlo, A. Galante, A.F. Grillo, Phys. Lett. B428 (1998) 166; Phys. Lett. B435 (1998) 175.

[13] I.M. Barbour, to appear in the proceedings of the Conference "QCD at finite baryon density", Bielefeld, April 1998.

[14] P.E. Gibbs, Phys. Lett. B172 (1986) 53.

[15] V. Azcoiti, G. Di Carlo, A. Galante, A.F. Grillo, V. Laliena, C.E. Piedrafita, Phys. Rev. D53 (1996) 5069. 